\title{
Publisher Correction: Exercise for improving age-related hyperkyphosis: a systematic review and meta-analysis with GRADE assessment
}

\author{
Matteo Ponzano ${ }^{1} \cdot$ Nicholas Tibert $^{1} \cdot$ Symron Bansal ${ }^{1} \cdot$ Wendy Katzman $^{2} \cdot$ Lora Giangregorio $^{1,3}$
}

Published online: 29 December 2021

○ International Osteoporosis Foundation and National Osteoporosis Foundation 2021

Publisher Correction: Arch Osteoporos

https://doi.org/10.1007/s11657-021-00998-3

The originally published article was the uncorrected proof; it has now been replaced by the corrected version. Springer Nature apologizes to the readers of the journal for not detecting the error during the publication process.

The original article has been corrected.

Publisher's Note Springer Nature remains neutral with regard to jurisdictional claims in published maps and institutional affiliations.

The original article can be found online at https://doi.org/10.1007/ s11657-021-00998-3.

Lora Giangregorio

lora.giangregorio@uwaterloo.ca

Matteo Ponzano

mponzano@uwaterloo.ca

Nicholas Tibert

ntibert@uwaterloo.ca

Symron Bansal

slimsym@hotmail.com

Wendy Katzman

wendy.katzman@ucsf.edu

1 Department of Kinesiology, University of Waterloo, Waterloo, Canada

2 Department of Physical Therapy and Rehabilitation Science, University of California San Francisco, San Francisco, USA

3 Schlegel-UW Research Institute for Aging, Waterloo, Canada 